\title{
Detección de instalaciones ilegales de agua potable con el uso de golpes de ariete
}

\section{Detection of illegal drinking water installations with the use of water hammers}

Recibido: febrero 10 de 2020 | Revisado: abril 26 de 2020 | Aceptado: mayo 15 de 2020

\author{
STEFAN ZieMENDORFF ${ }^{\mathrm{I}}$ \\ SWEN SEWERIN ${ }^{2}$ \\ JOHn MONTERO3
}

1 Autor corresponsal: Programa de Modernización y Fortalecimiento del Sector Agua y Saneamiento - PROAGUA 11 , sziemendorff@gmail.com

2 Universidad de Bonn. Alemania

3 Universidad Nacional Santiago Antúnez de Mayolo, HUaraz (UNASAM). Perú

\begin{abstract}
Resumen
En una anterior investigación se ha demostrado que una de las formas de instalaciones ilegales, destinado al hurto de agua potable y llamado comúnmente bypass, resulta con frecuencia imposible de detectar con la aplicación de los métodos conocidos. Por ello se presenta en este artículo una técnica nueva - el uso de golpes de ariete con la medición y análisis de transientes hidráulicos, el cual puede en la mayoría de los casos indicar si existe o no un bypass ilegal. Para ello se analizan los principales factores que influyen a la aplicabilidad y precisión del método, así como sus limitaciones de aplicación con el uso de ensayos de campo y simulaciones computarizadas.
\end{abstract}

Palabras clave: Agua potable, instalaciones ilegales, golpes de ariete, transientes hidráulicos

\begin{abstract}
In a previous investigation, it has been shown that one of the forms of illegal installations intended for clandestine appropriation of drinking water and commonly called bypass, resulting frequently impossible to detect with the application of known methods. For this reason a new technique is presented in this article - the use of water hammers with the measurement and analysis of hydraulic transients, which in most cases indicate whether or not there is an illegal bypass. To this end, the main factors that influence the applicability and precision of the method, as well as its application limits, are analyzed with the use of field trials and computer simulations.
\end{abstract}

Key words: Drinking water, illegal installations, water hammers, hydraulic transients

(C) Los autores. Este artículo es publicado por la Revista Campus de la Facultad de Ingeniería y Arquitectura de la Universidad de San Martín de Porres. Este artículo se distribuye en los términos de la Licencia Creative Commons Atribución No-comercial - Compartir-Igual 4.0 Internacional (https://creativecommons.org/licenses/ CC-BY), que permite el uso no comercial, distribución y reproducción en cualquier medio siempre que la obra original sea debidamente citada. Para uso comercial contactar a: revistacampus@usmp.pe. 


\section{Introducción}

En tiempos de creciente escasez del líquido elemento, los esfuerzos de las entidades prestadoras de los servicios de agua potable de disminuir sus niveles de Agua No Facturada (ANF) están en aumento, por un lado orientado en disminuir el nivel de las pérdidas físicas con un mejor control preventivo y correctivo de las fugas y por otro lado en disminuir las pérdidas aparentes o comerciales con el incremento de la micromedición y la mejora de la eficiencia del parque de medidores, evitando así el subregistro de los consumos.

Sin embargo, aparte de las fugas y el subregistro, existe un tercer factor de importancia en el ANF a nivel mundial: El hurto de agua potable, también llamado consumo no autorizado o coloquialmente clandestinaje del agua. Por lo oculto de esta práctica es muchas veces difícil de estimar su importancia, pero queda claro que sus efectos van más allá de las pérdidas directas que causa, ya que afecta también la sostenibilidad económica-financiera de las entidades prestadoras $y$, por lo antitécnico de las instalaciones ilegales, es una causa importante de las fugas de agua.

El hurto de agua potable se realiza de muchas formas diferentes (Ziemendorff et al. 2020) como a través de la manipulación de medidores de consumo ( Ziemendorff 2020), la reapertura (o reconexión) ilegal de conexiones previamente cerradas (Ziemendorff et al. 2018 y 2019), por ejemplo por la falta de pago del servicio y a través de instalaciones ilegales permanentes, con frecuencia también llamadas clandestinas o fraudulentas, las cuales se dividen por su forma de ser instaladas en tres, como se puede apreciar en la siguiente Figura 1.

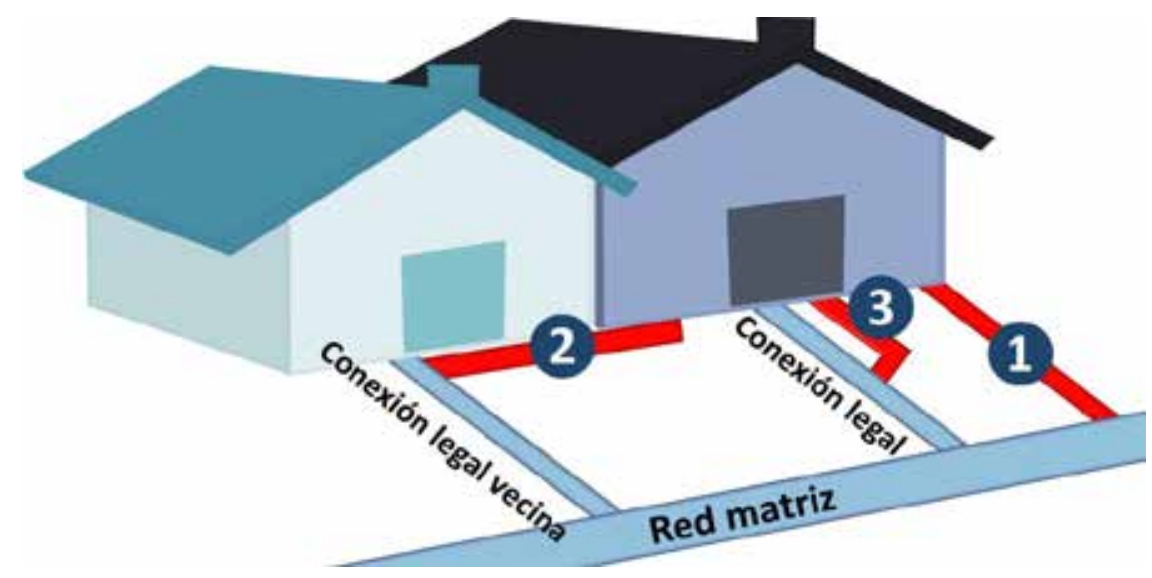

Figura 1. Formas de instalaciones ilegales: (1 Conexión directa a la red pública, 2 Derivación de una conexión vecinal, 3 Derivación a la propia conexión legal - Bypass

De una investigación anterior (Ziemendorff et al. 2020), en la cual se compararon diferentes técnicas de localización de instalaciones ilegales, se llegó a la conclusión de que estas son efectivas para la detección de las primeras dos formas, pero en muchos casos limitadas para la detección de bypasses, específicamente por las siguientes razones:

1. La señal del Georadar no puede penetrar a través de la caja del medidor, debajo de la cual se encuentran frecuentemente los bypass, También es frecuente no poder distinguir un tubo (ilegal) que se encuentra justo por debajo, encima o al costado de otro tubo (legal). Ello 
es importante porque los bypasses son frecuentemente ejecutados de esta manera.

2. Las cámaras endoscópicas con frecuencia no pueden ser introducidas en conexiones domiciliarias por la presencia de codos de $90^{\circ}$, las cuales presentan un obstáculo para el paso del equipo de inspección por video.

3. El método electroacústico detecta la presencia de la tubería legal o ilegal en el subsuelo desde la superficie, pero es casi imposible distinguir entre ambos en cuando estas se encuentran sobrepuestas o muy cercanas, como es característico de los bypass.

Por ello en el presente artículo se investigan los alcances y límites de una nueva alternativa, la cual puede servir para superar esta limitante y complementar de esta manera los métodos antes mencionados.

\section{El golpe de ariete y sus implicancias en el método propuesto}

Para comprender, adecuadamente, el método propuesto para la detección de conexiones ilegales tipo bypass, es necesario conocer bien lo que es el golpe de ariete tal como lo señala (Franzini et al., 1999). El golpe de ariete es la disminución brusca de la velocidad de un líquido en una tubería debido al movimiento (cierre/apertura) de una válvula, ocasionando una serie de ondas cinéticas que se transforman en ondas de compresión y viceversa, provocando que la masa líquida (en nuestro caso el agua) se comporte como un resorte (1 999:358); dichas oscilaciones pueden registrarse como curvas de presiones a lo largo de intervalos de tiempo cortos.

El golpe de ariete o transitorio es el fenómeno físico base para la aplicación del método propuesto para la detección de bypasses ilegales, puesto que al cerrar la válvula de control de una conexión de agua potable sometida a presión, se van generar ondas de presiones de diversas magnitudes, las mismas que pueden ser registradas en un dispositivo datalogger de modo que se puedan establecer un patrón de oscilación para una conexión sin bypass y otro para una conexión con bypass.

\section{Formas de bypasses y la posibilidad de su detección}

Hay diferentes formas de colocar un bypass ilegal a la conexión propia legal y las formas de detectarlo dependen de cómo fue colocado. Específicamente depende de:

1. Si el bypass se conecta al mismo circuito de agua interno del predio.

2. $\mathrm{Si}$ en el momento de realizar la inspección de la conexión sospechosa se está realizando algún consumo.

En el primer caso, la detección del consumo ilegal es relativamente simple, porque saldría agua desde el predio aún si fue cortado previamente el servicio. Para distinguir si se trata agua de fuente propia o de un reflujo de un tanque de almacenamiento interno se deben comparar las características del agua que sale del predio con aquel de la red pública en este punto. Para ello se puede comparar la temperatura del agua, la cual es normalmente mayor si sale de un tanque que de la red pública, la concentración del cloro residual, muchas veces ausentes en fuente propias, las características organolépticas, pero sobre todo la presión la cual si es igual a la de la red pública en este punto será un indicador claro de la existencia de un bypass. Sin embargo en la práctica muy pocas veces se puede detectar un bypass con ese método, sea porque el bypass no está conectado al 
circuito interno, sea porque cuenta con una llave cerrada al momento de la inspección o porque el predio cuenta con una válvula de antirretorno.

En el segundo caso, basta con cerrar el servicio legal por un corto tiempo, y comparar la presión de esta conexión sospechosa con la presión normal en este momento y en este punto (se puede tomar como referencia una conexión vecina), porque al momento de realizar un consumo a través del bypass, la presión variará considerablemente debajo del nivel normal. Igualmente es raro detectar un bypass de esta forma - por un lado porque el tiempo de uso del servicio de agua es normalmente corto y por el otro lado porque la presencia de personal del operador puede alertar al hurtador de no usar el servicio en el preciso momento en el cual se realiza la inspección de su conexión.

Como ambos métodos solo llevarán a hallazgos muy fortuitos de bypasses, se requiere de una forma capaz de detectarlos aún si no hay un reflujo de agua desde el predio sospechoso y en momentos que no se realiza consumo a través del bypass. Los autores del presente artículo propusieron en el 2017 a la Cooperación
Alemana al Desarrollo GIZ la realización de un proyecto piloto, destinado a probar la factibilidad de un nuevo método. Este consiste en provocar un golpe de ariete en la conexión sospechosa de tener un bypass y medir los transientes con un datalogger de presión y analizar la data obtenida presenta singularidades que permitan predecir si de la conexión se deriva una toma de agua.

Este método ya ha sido propuesto e investigado para la detección de fugas de agua, aprovechando el comportamiento diferencial de los transientes en tuberías con o sin fugas (Brunone et al. 2004; Shucksmith et al. 2012). Por ello, se infirió que puede haber un comportamiento igualmente diferente entre el desarrollo en el tiempo de transientes en conexiones sin bypass, frente a conexiones de características iguales que sí cuentan con un bypass.

\section{Materiales y método del ensayo piloto}

Para averiguar cómo funciona el método en campo y si en principio podrá dar resultados favorables se instaló en la ciudad de Moyobamba (Perú) un circuito de ensayos, el cual tenía las siguientes características (Figura 2):

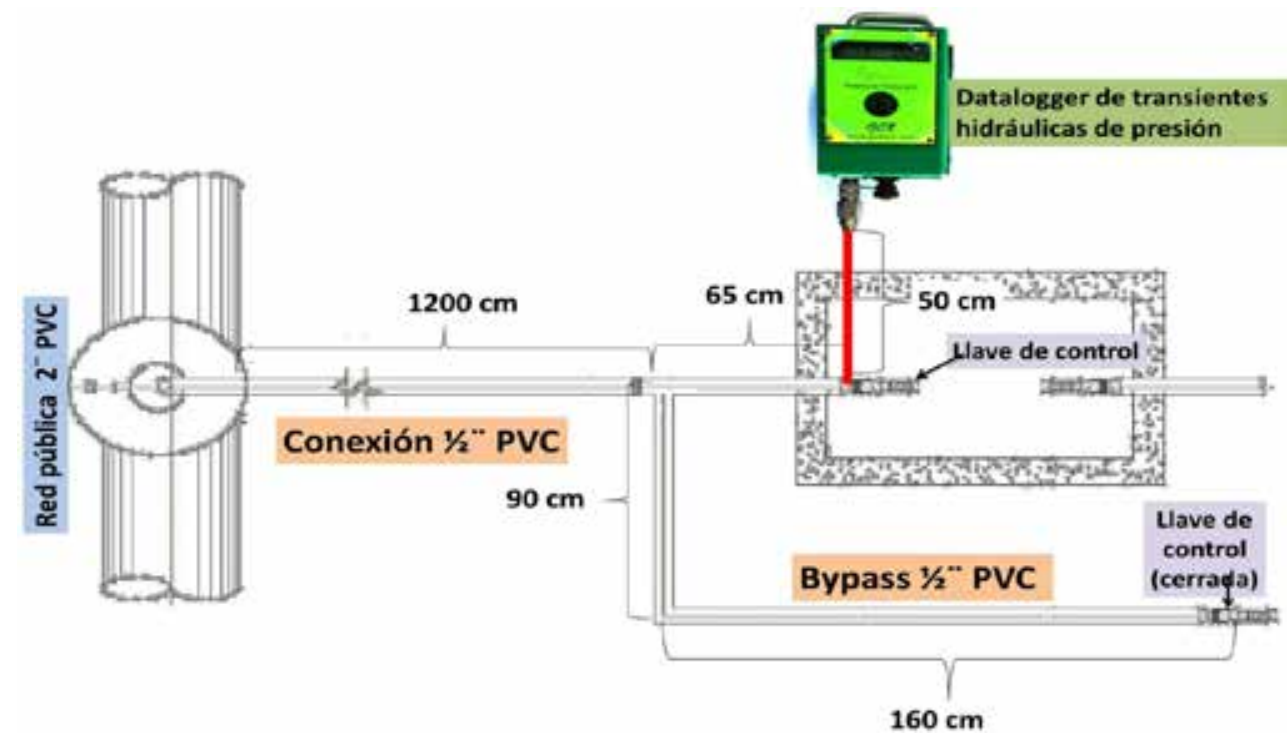

Figura 2. Esquema del ensayo piloto 
- Material de la tubería: PVC

- Diámetro de la conexión y del bypass: DIN 15 (1/2")

- Longitud de la conexión: $12 \mathrm{~m}$

- Distancia de la toma del bypass hasta la llave de control: $65 \mathrm{~cm}$

- Longitud del bypass: $250 \mathrm{~cm}$

- Tipo de llave de control: Tipo bola

- Presión del agua: $20 \mathrm{mca}$ (metros de columna de agua $=28,5$ PSI) aprox.
Luego se conectó un Datalogger de presiones transientes a través de una manguera (accesorio del equipo) de 50 centímetros de largo y $6 \mathrm{~mm}$ de diámetro entre la llave de control (también llamada válvula de control) delante del medidor y la red pública, preferiblemente lo más cercano posible a la llave. El Datalogger registra los cambios en la presión hasta un máximo de 1000 veces por segundo. En nuestro caso, se fabricó un pequeño adaptador de bronce que facilitó su rápida instalación (Figura 3).

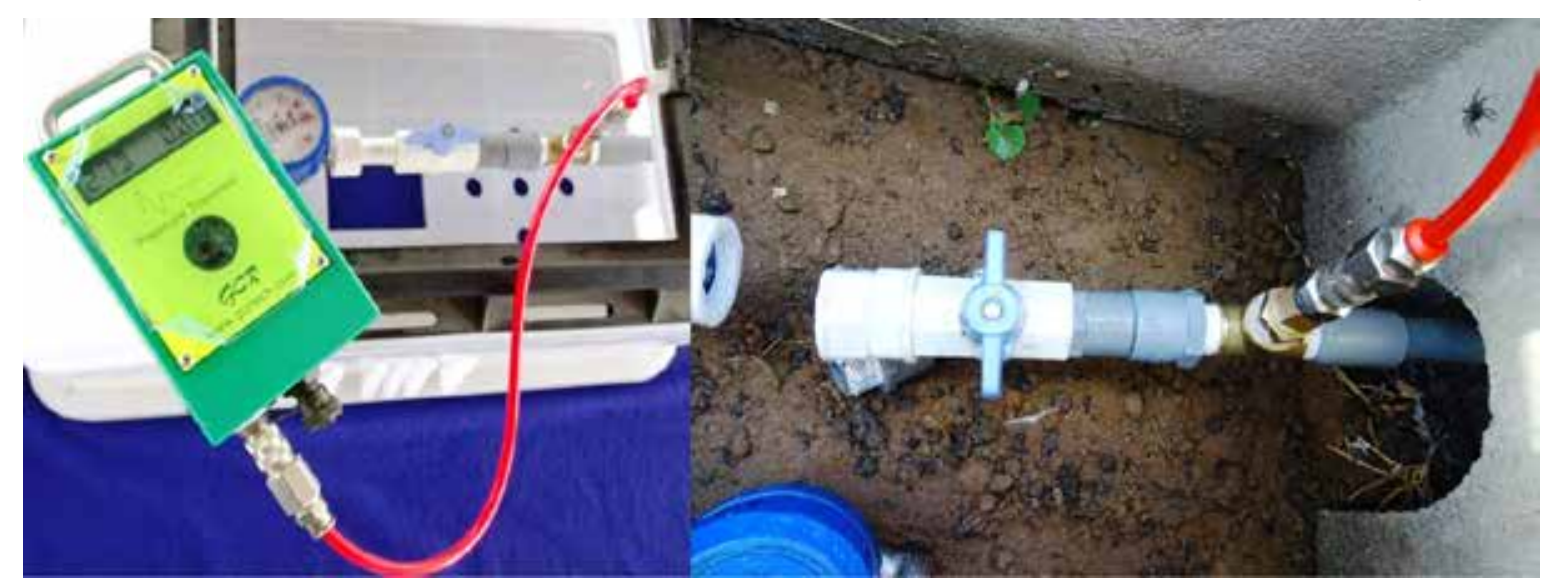

Figura 3. Izquierda: Datalogger instalado; Derecha: Manguera delante de la llave de control instalada con un adaptador de bronce.

El método del ensayo piloto fue el siguiente:

1. Se realizó primero una serie de ensayos con bypass. Previo al ensayo es necesario de instalar el Datalogger.

2. Para ello, se dejó primero la llave de control (también llamada válvula de cierre) abierta y luego se procedió a cerrarla de golpe (manualmente). De esta forma se procedió siete veces en forma consecutiva, siempre esperando un medio minuto entre cada ensayo.

3. Posteriormente, se anuló el bypass y los ensayos siguientes fueron ejecutados en la misma conexión.

4. Luego se procedió de la misma forma, dejando primero la llave de control abierta y cerrándola de golpe para realizar otra serie de siete ensayos iguales, pero esta vez sin el bypass para contar con datos comparativos.

5. Finalmente, los datos fueron descargados. Cabe indicar que el datalogger fue programado para que registre 100 datos por segundo (no los 1000 datos, que es su capacidad máxima) a propuesta del experto de la empresa proveedora del equipo.

\section{Resultados del ensayo piloto}

Debido a que la llave de control fue cerrada manualmente, los resultados de los siete ensayos con bypass y los siete sin este presentaron ciertas variaciones pero con un padrón casi uniforme, verificándose que hay dos variaciones principales:

1. El primer golpe de presión era más 
fuerte en los ensayos con bypass que en aquellos sin bypass.

2. En los ensayos con bypass el número de oscilaciones de la presión es mucho mayor que en los ensayos sin bypass.

Para ilustrar ambos efectos, se ha sobrepuesto en la siguiente Figura 4 a manera de ejemplo una serie de datos de los ensayos sin bypass con una con bypass, para un intervalo de tres segundos que inicia poco antes del cierre de la llave de control. Cabe indicar que el gráfico es ejemplar en el sentido que se ha escogido de los ensayos los resultados que más se acercan al promedio del total de los ensayos.

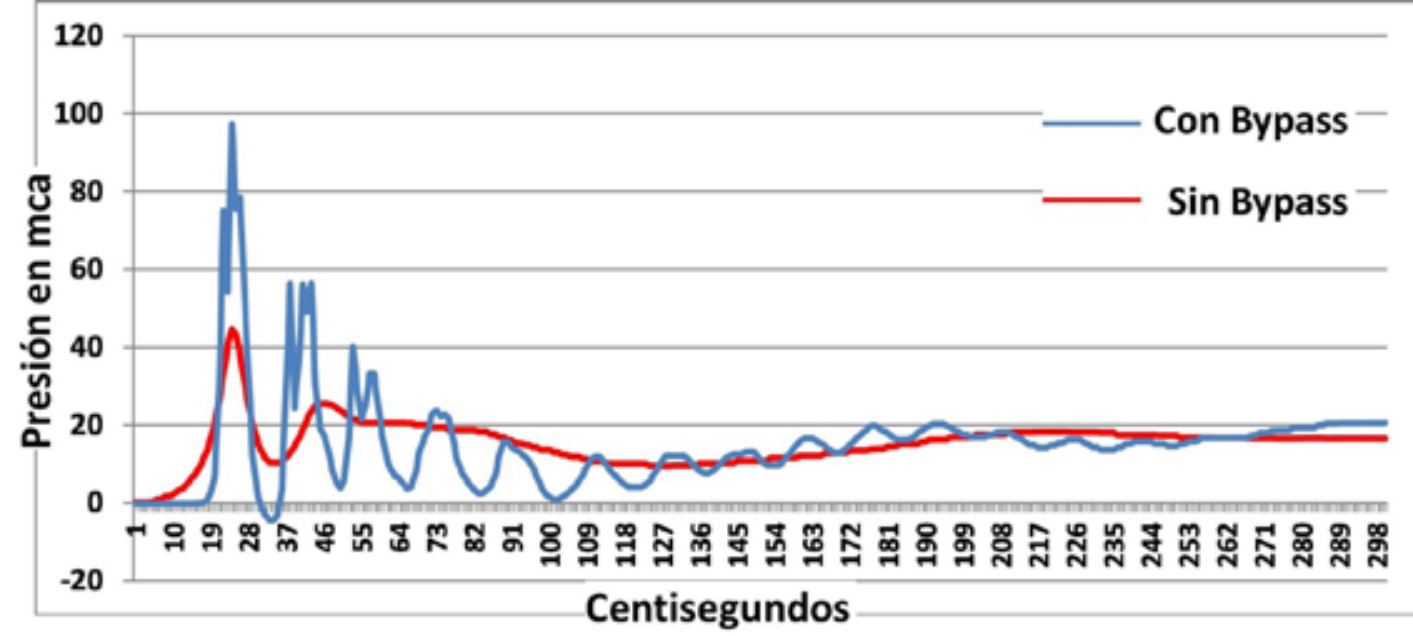

Figura 4. Resultado ejemplar de los ensayos con y sin bypass

Como se nota, el resultado es favorable en el sentido que el método puede servir para detectar bypasses ilegales. Este primer resultado; sin embargo, es válido solamente para las condiciones específicas del ensayo.

\section{Materiales y métodos de las simulaciones}

Para conocer si el método tiene un uso práctico para la detección de bypass, más allá de las condiciones específicas de los ensayos realizados, era necesario ver cómo varían los resultados acorde a las condiciones. Específicamente lo que se quería evaluar son las variables siguientes:

(1) Influencia de la velocidad de cierre de la llave de control, para recomendar eventualmente válvulas de cierre magnético rápido.

(2) Influencia de la longitud y del material de la manguera de alimentación del Datalogger, para conocer si y por cuanto se puede mejorar los resultados acortando esta manguera o reemplazándola con un material más rígido.

(3) Influencia de la presión: Para conocer a partir de que presión en la red el método es aplicable.

(4) Influencia del diámetro: Para conocer si el método funciona también (quizás mejor) en tuberías de mayor diámetro $(3 / 4 "-2 ")$

(5) Influencia de la distancia de la toma del bypass hasta la llave de control, para conocer si el método es apto para todos tipos de bypass.

(6) Influencia de la longitud del bypass, desde la toma en la conexión, hasta el punto terminal en el predio que usa ilegalmente el agua.

Debido a la gran cantidad de combinaciones posibles ya no era factible de realizar la investigación mediante el método del ensayo físico. Por ello se recurrió 
a softwares especializado para el análisis de golpes de ariete y transientes, con las cuáles es posible simular una gran cantidad de variaciones en poco tiempo. El objetivo de estas simulaciones era conocer los alcances y límites del método, así como el de dar algunas indicaciones para su correcto y óptimo uso.

\section{Resultados de las simulaciones}

Influencia de la velocidad de cierre de la llave: La velocidad del cierre de la llave influye directamente en la magnitud del golpe de ariete, cuya generación es necesaria para poder distinguir entre conexiones con un bypass y conexiones sin él. A mayor velocidad de cierre - el golpe es mayor y por ello los resultados son de más fácil interpretación, por lo cual cabía la posibilidad de mejorar los resultados con el uso de una válvula de cierre magnético. Ello además permitiría estandarizar la velocidad de cierre y hacer, en teoría, los resultados de más fácil comparación.

Para averiguar el grado en que influye la velocidad de cierre se procedió primero tomar el tiempo de velocidad de cierre manual, el cual varía en un rango de $0.15 \mathrm{~s} \mathrm{a}$ $0.20 \mathrm{~s}$, tomando en cuenta que la velocidad aumenta en el proceso de cierre.

Las simulaciones dieron como resultado que el golpe de presión no aumenta en forma perceptible si se aumenta la velocidad de cierre manual al doble y comienza a bajar recién en forma importante cuando se disminuye la velocidad de cierre, aumentando del tiempo de cierre por encima de $0.40 \mathrm{~s}$. Ello quiere decir que el empleo de una válvula magnética de cierre más rápido y estandarizado no es necesario, ya que el rango de velocidad de cierre manual se encuentra dentro lo deseable para lograr resultados óptimos.

\section{Influencia de la longitud y del material} de la manguera de alimentación del datalogger: Como la idea base del método es poder distinguir conexiones con bypass de aquellas sin él, aparenta lógico de tratar de disminuir aquellos factores que puedan influir los resultados negativamente. Por ello se evalúo la posibilidad que la manguera de alimentación del Datalogger (la manguera roja que se nota en la Figura 3) podría cortarse o reemplazarla por otro material. Con esto se podría, en teoría, lograr que la distancia a recorrer por las ondas de presión hasta el punto donde se mide su fuerza (el Logger) sea menor y los golpes y sus respetivos rebotes podrán propagarse mejor. La manguera proveída junto con el Datalogger tenía una longitud de $50 \mathrm{~cm}$, un diámetro de $6 \mathrm{~mm}$ y de material plástico-flexible.

Las simulaciones dieron como resultado que el reemplazo del material por uno más rígido (hierro dúctil) no varía los resultados de manera perceptible, mientras si hay un impacto muy ligero de la longitud de la manguera de alimentación. Si esta se acorta de $50 \mathrm{~cm}$ a $20 \mathrm{~cm}$ el golpe de presión varía en apenas un $3 \%$. Tomando en cuenta que acortar la longitud de la manguera presenta serios desconvenientes para el operador del Datalogger, al incrementar la dificultad de colocarlo en la llave, no resulta conveniente variar la confección de la manguera como viene del fabricante.

Influencia de la presión: Según las simulaciones realizadas con presiones entre 2 mca (2.8 PSI) y $120 \mathrm{mca}$ (171 PSI), es decir dentro de todo el rango que se presenta en el campo, el método funcionará, porque sólo cambian los valores absolutos de las presiones, más no su tendencia.

Influencia del diámetro: Se han efectuado los mismos simulacros en 
tuberías de 1/2", 3/4", 1" y 2" con el resultado que el golpe de ariete inicial y sus respetivos rebotes son mayores a mayor diámetro. Por ello se concluye que el método no es solamente apto para conexiones de mayor diámetro (al diámetro del ensayo de campo) sino que inclusive funcionará mejor en estos. Ello es de suma importancia, porque si bien conexiones con diámetros mayores no son frecuentes, el consumo realizado en estas es normalmente mucho mayor.

Influencia de la distancia de la toma del bypass hasta la llave de control: De todos los parámetros aquí analizados es aquel que presenta la mayor influencia en los resultados. En específico se ha encontrado a través de las simulaciones que los bypasses muy cercanos a la llave y por ende más alejados de la red son de más fácil detección. Específicamente se encontró que no es tanto la distancia absoluta sino la relación distancia a la llave/distancia a la red es que impacta a los resultados en el sentido que bypasses que se encuentran en la última tercera parte de la conexión hacia la red son los más difíciles de distinguir de conexiones sin bypass.

En la realidad los bypasses que son encontrados continuamente en el campo por los operadores de servicios de agua se hallan a poca distancia de la caja portamedidor y con ello de la llave y raras veces superan el metro y medio de distancia.

Por ello, concluimos que el método solo podrá dejar dudas sobre la probable existencia de un bypass o no para el caso de conexiones muy cortas (de uno o dos metros), las cuales, si bien no son la mayoría de las conexiones de agua potable, representan variando de sitio a sitio un grupo importante de conexiones. Ello no quiere decir que es imposible de encontrar con el método bypasses en conexiones cortas, sino que la interpretación de los resultados se dificulta de tal forma que en ciertas ocasiones se puede tener falsos positivos y en otras obviar un bypass.

\section{Influencia de la longitud del bypass:} Ello se refiere a la longitud del bypass desde la toma en la conexión, hasta el punto terminal en el predio que usa ilegalmente el agua. Las simulaciones dieron como resultado que la longitud prácticamente no influye en los resultados - es decir un bypass que es detectable con el método con un bypass de una longitud de 1 metro es igualmente detectable si tuviera 50 metros o más. Ello es de suma importancia, porque la longitud del bypass si puede variar mucho en la realidad.

\section{Discusión}

Los resultados de los ensayos de campo, combinado con aquellos de las simulaciones muestran un método para la detección de bypasses y derivaciones ilegales de fácil aplicación y pocas limitaciones. A este resultado, sumamente positivo, que pone una nueva herramienta muy útil en manos de las entidades prestadora hay que agregar sin embargo unas consideraciones adicionales.

La primera es acerca de los límites de aplicación por la presión del agua. Si bien los ensayos mostraron que el método funciona también con presiones muy altas es necesario mencionar que golpes de ariete, especialmente en sistemas antiguos pueden tener el efecto colateral de dañar la tubería y causar fugas. Para mitigar el impacto de ello, es posible tomar tres medidas:

1. Limitar la cantidad de golpes de ariete al aplicar el método solo en aquellas conexiones que ya fueron identificadas como de consumos sospechosamente 
bajos (para los métodos estadísticos que pueden servir (Alva et al. 2020).

2. Aplicar el método en horarios de más baja presión - ello debido a que en el transcurso del día las presiones varían. Normalmente los horarios de menor presión son conocidos por las entidades prestadores de servicio.

3. Regular la presión hacia abajo en aquellas zonas donde se aplica el método, mientras se aplica, ello se facilitará cuando se cuente con sistema de abastecimiento de agua sectorizado.

La segunda consideración es acerca de la exactitud de los datos obtenidos mediante las simulaciones computarizadas. Resultó en la práctica imposible recrear en un $100 \%$ las condiciones reales, por ejemplo las uniones entre dos tuberías en la realidad nunca son de tal perfección como asume el modelamiento del software o cuando se presentan intersecciones o doblamientos en la tubería etc. Así no se pudo obtener mediante los simulacros datos muy precisos, sino una idea bastante clara en qué dirección y con qué magnitud influye cual factor. Los resultados son presentados en este sentido, dejando algunas dudas que solamente pueden ser solucionados con pruebas más amplias de campo bajo condiciones diversas. En específico les queda a los autores del presente texto la duda si bajo condiciones reales, será realmente posible detectar un bypass con el método cuando la presión es tan baja como $2 \mathrm{mca}$ (2.8 PSI) o si, por las imperfecciones implícitas en cualquier sistema de agua, especialmente cuando este es de mayor antigüedad, el golpe de presión se disipa tan rápidamente que ya no permite hacer la detección.

La consideración final es que el método presentado es, por lo menos por el momento, un método de detección y no de localización de instalaciones ilegales. Ello quiere decir que en caso de una detección será conveniente de iniciar las excavaciones para descubrir las instalaciones ilegales por donde se encuentran con mayor probabilidad - esto es cerca de la caja portamedidor. Quedaría por averiguar si con más amplias experiencias se puede ganar mejores datos que permiten interpretar los resultados de tal forma que también permitan con algún grado de precisión la ubicación de la toma ilegal. Ello es teóricamente factible, ya que hemos visto que la distancia de la toma a la llave/al datalogger si afecta a los resultados.

\section{Conclusiones}

1. Con los resultados presentados es posible afirmar que se cuente con un nuevo método de detección de dos tipos de instalaciones ilegales - derivaciones de una conexión vecina y bypasses.

2. El método puede ser usado tal como descrito en el ensayo piloto, quiere decir con el cierre manual de la llave de control y la manguera alimentadora prevista por el fabricante.

3. El método no presenta mayores limitaciones en lo que al diámetro de la conexión y la presión del agua se refiere, aunque en este último caso se debería tratar los resultados de las simulaciones con precaución.

4. La única dificultad que se pudo identificar con las simulaciones se refiere a los casos de conexiones muy cortas, porque en estas pueden existir con frecuencia bypasses muy cercanos a la red que solo pueden ser detectadas con dificultad con el nuevo método descrito.

\section{Recomendaciones}

1. En muchos casos el método presentado con Dataloggers de presión es el único 
capaz de detectar bypasses y por ello se convierte en un excelente complemento de otros métodos como el método electro acústico o el Georadar. Uno de estos métodos deberían ser a la vez el complemento a Dataloggers de presión, porque al contrario de este pueden detectar conexiones ilegales directas en la red pública.

2. En sitios con alta presión del agua y redes antiguas se debería aplicar el método con cautela. Esto es usarlo solo cuando haya una sospecha previa y en horario de menor presión del agua o, de ser técnicamente factible, regular la presión hacía bajo. De esta manera, se puede evitar que los golpes de presión causen daños (fugas) en las conexiones y redes.
3. Debido a que las simulaciones computarizadas realizadas no pueden reemplazar la experiencia de campo, los resultados deben ser confirmados y reajustados en un futuro, tomando en cuenta estas experiencias prácticas. La presente publicación tiene en este sentido la finalidad de animar a las entidades operadoras de servicios de saneamiento de aplicar el método para obtener datos más fidedignos.

4. Será de interés especial, si, como parte de los resultados de campo, se podría ampliar la investigación acerca de la factibilidad de usar el método presentado no solamente para la detección sino también hasta cierto grado para la localización de las tomas ilegales.

\section{Agradecimientos}

Queremos agradecer a CTM TECTROL SAC, Perú por la provisión de los equipos, a la CONSULAQUA Hamburg GmbH, Alemania con el apoyo de simulaciones computarizadas, así como al equipo comercial de la EPS Moyobamba S.A. (Perú) para el apoyo logístico en los ensayos de campo.

\section{Referencias}

Alva, T. \& Ziemendorff, S. (2020). Manuales de gestión comercial. Manual 4: Prevención y detección de hurtos. Lima: OTASS.

Brunone, B.; Ferrante, M.; Covas, D.; Ramos H. \& Betâmio de Almeida, A. (2004). Detecting leaks in pressurised pipes by means of Transients. Journal of Hydraulic Research, 42(1), 105109.

Franzini J. \& Finnemore J. (1999). Mecánica de Fluidos con Aplicaciones en Ingeniería. Madrid: McGraw Hill.
Shucksmith, J.; Boxall, J.; Staszewski, W.; Seth, A. \& Beck, S. (2012). Onsite leak location in a pipe network by cepstrum analysis of pressure transients. Journal American Water Works Association, 104(8), E457-E465.

Ziemendorff, S. \& Kersting, M. (2020). Comparación de tecnologías para la localización de conexiones ilegales de agua potable. CAMPUS, 25(29), 163-176. DOI: doi.org/10.24265/ campus.2019.v25n29.12 
Ziemendorff, S. y Perfecto O. (2017). Comparación de técnicas de corte simple del servicio de agua potable. CAMPUS, Vol. 22, $\mathrm{N}^{\circ}$ 24: 229250. DOI: doi.org/10.24265/ campus.2017.v22n24.08

Ziemendorff, S.; Perfecto O.; Mestanza, S. y Chuquizuta, C. (2018). Comparación de técnicas de corte drástico del servicio de agua potable. CAMPUS, Vol. 23, $\mathrm{N}^{\circ} 25$ : 67-86. DOI: doi.org/10.24265/ campus.2018.v23n25.06

Ziemendorff, S. (2020). Medidasindicativas y preventivas contra la manipulación fraudulenta de medidores de agua. Tecnologia y Ciencias del Agua, Vol. $11, \mathrm{~N}^{\circ} 4$. 
